\title{
ARTICLE OPEN \\ 3D non-isothermal phase-field simulation of microstructure evolution during selective laser sintering
}

\author{
Yangyiwei Yang $\mathbb{D}^{1}$, Olav Ragnvaldsen ${ }^{2}$, Yang Bai ${ }^{1}$, Min $\mathrm{Yi}^{3}$ and Bai-Xiang Xu (D)
}

During selective laser sintering (SLS), the microstructure evolution and local temperature variation interact mutually. Application of conventional isothermal sintering model is thereby insufficient to describe SLS. In this work, we construct our model from entropy level, and derive the non-isothermal kinetics for order parameters along with the heat transfer equation coupled with microstructure evolution. Influences from partial melting and laser-powder interaction are also addressed. We then perform 3D finite element non-isothermal phase-field simulations of the SLS single scan. To confront the high computation cost, we propose a novel algorithm analogy to minimum coloring problem and manage to simulate a system of 200 grains with grain tracking algorithm using as low as 8 non-conserved order parameters. Specifically, applying the model to SLS of the stainless steel 316L powder, we identify the influences of laser power and scan speed on microstructural features, including the porosity, surface morphology, temperature profile, grain geometry, and densification. We further validate the first-order kinetics of the transient porosity during densification, and demonstrate the applicability of the developed model in predicting the linkage of densification factor to the specific energy input during SLS.

npj Computational Materials (2019)5:81; https://doi.org/10.1038/s41524-019-0219-7

\section{INTRODUCTION}

Selective laser sintering (SLS) is a typical additive manufacturing (AM) process meant for rapid prototyping and tooling. ${ }^{1-5}$ During SLS, a desired geometry is built by sequentially layer-by-layer powder spreading and subsequent sintering driven by laser scan. ${ }^{6,7}$ To be distinguished with the other laser-based powder bed additive manufacturing known as selective laser melting (SLM), there is no significant melting phenomenon during SLS. Whereas the temperature is sufficiently high for particles to bind together through sorts of mechanisms, leading to the products with relatively high porosity. ${ }^{5,8,9}$ Because of these characteristics, SLS has been applied for the industrial production of individually designed components made of organic polymers, ceramics, and metallic alloys which have relatively high melting or transition temperatures. ${ }^{1,5,10,11}$ It also shows the possibility to produce porous biomaterials, especially medical scaffold and bones, ${ }^{12-15}$ and functional materials through SLS. ${ }^{10,16,17}$

Although the procedure of SLS is conceptually simple, the underlying physics are complex and cover a broad range of time and length scales. Since its birth, ${ }^{18}$ identification and comprehension of those phenomena during SLS and their effects on the final product are crucial for the successful manufacturing, which highly relies on trial-and-error and even sort of empirical knowl-

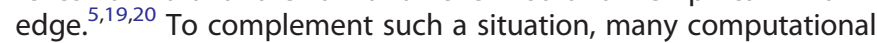
works have been performed. On the one hand, there is strong interest in thermal simulation revealing the intricate heat transfer, which serves as the thermodynamic indicator for other physical processes. However, due to the difficulties in modeling the interactive topography evolution of the powder bed, simplifications are often employed in simulating the thermal profile during the powder bed based AM. For instance, the powder bed is frequently assumed to be a homogeneous substance material with effective thermal properties. ${ }^{6,21-25}$ There are also studies on the particle scale, ${ }^{23,26,27}$ but the heat transfer and the particle shape change are not fully coupled. Besides, the thermal effect from laser-powder interaction is often formulated as an equivalent internal heat source. ${ }^{7,28-30}$ Alternatively, the laserpowder interaction can also be treated via ray tracing, ${ }^{31}$ but the computation is extremely expensive. On the other hand, simulating the microstructure evolution plays an essential role since it is directly related to mechanical properties of the final products like tensile strength, ductility, and fracture toughness. Up to now, however, simulation of the microstructure evolution still remains a great challenge if influences from all aspects are considered. During scanning, there is a drastic difference in the thermal conditions among particles due to different exposure to the laser beam. Some of the particles are even partially melted during the process. ${ }^{5,32}$ Therefore, the binding mechanism for certain particles/grains may vary from the solid-state sintering to the liquidstate sintering, and even melting-solidification depending on the intensity of its partial melting. ${ }^{8,32-34}$ For the same reason, very high temperature gradients and cooling rates also exist, ${ }^{7,35}$ which make the mechanism of grain coalescence and coarsening during SLS deviate from that in conventional isothermal sintering.

In recent decades, the phase-field method has been utilized to simulate the microstructure evolution during sintering-related techniques, thanks to its ability to model the complex patterns with no need of tracking the position of the surface and interface

\footnotetext{
${ }^{1}$ Mechanics of Functional Materials Division, Institute of Materials Science, Technische Universität Darmstadt, 64287 Darmstadt, Germany; ${ }^{2}$ Department of Materials Science and

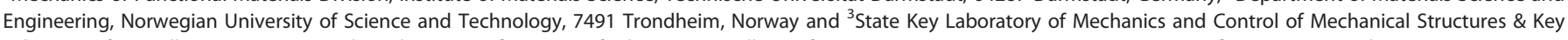

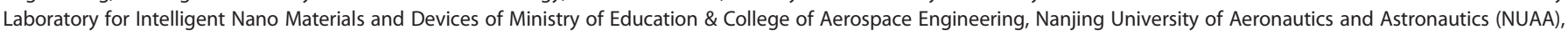
210016 Nanjing, China

Correspondence: Min Yi (yimin@nuaa.edu.cn) or Bai-Xiang Xu (xu@mfm.tu-darmstadt.de)
}

Received: 10 February 2019 Accepted: 10 July 2019

Published online: 06 August 2019 
explicitly. One of the earliest phase-field sintering models was suggested by Wang et al., who utilized a conserved density field and a set of non-conserved orientation fields. ${ }^{36,37}$ Based on this model, some featured details, such as the dihedral angle, neck growth, shrinkage, and the grain growth kinetics with porosity, have been predicted and validated by experiments. ${ }^{38-40}$ Zhang et al. ${ }^{41}$ adopted this isothermal model to investigate the necking among several powders during the SLS. Nevertheless, the mentioned simulations are for the isothermal case, while the heat transfer and the coupled non-isothermal microstructure evolution are not addressed, and thus cannot be applied for the study of SLS. Lu et al. $^{42}$ presented two-dimensional (2D) phase-field simulations on laser-powder bed fusion using a simplified free energy functional based on interpolation functions of temperature. They reproduced certain important features including porosity and grain size changes w.r.t. specific energy density. However, the thermodynamic consistency of the used model is not shown. In particular, their free energy functional does not include thermal and configurational entropy contributions, and thus the temperature-dependency of the surface/grain-boundary energy is not regarded. Moreover, 3D simulations are necessary because of the spatial feature of the laser beam, and able to provide more accurate information on surface morphology and grain geometry.

In this work, we perform 3D non-isothermal phase-field simulations to investigate the microstructure evolution during the SLS process. The model is constructed from the entropy level following our latest work, ${ }^{43}$ explicitly considering the contributions from thermal, configurational, and gradient terms. We then obtain a free energy density functional with temperaturedependent heat, local, and gradient terms through Legendre transformation, which is frequently used in thermodynamics analysis for non-isothermal conditions. ${ }^{36,44,45}$ Such formulation is able to reproduce the temperature-induced inhomogeneity of surface and grain-boundary energies, and hence their influences on kinetics, e.g. the mass transfer induced by inhomogeneity of surface. ${ }^{43}$ Apart from this, after thermodynamics analysis, we obtain simultaneously modified kinetics for order parameters (OPs), including the mass transfer and grain growth under the non-isothermal conditions, and a heat transfer equation coupled with microstructure evolution. We also consider the laser-powder interaction including absorption, reflection, and penetration of the laser radiation, and the partial melting contribution on the mass transfer. A scenario with a conserved order parameter as well as a series of non-conserved ones is utilized to represent the microstructure evolution during SLS. The discrete element method (DEM) is used to generate the geometry of the initial powder bed. In order to reduce the computation cost associated with a large amount of degree of freedoms, the Welsh-Powell algorithm for the minimum coloring problem (MCP) is for the first time applied to optimize the assignment of OPs. As examples, we perform phase-field simulations on the SLS processing of stainless steel $316 \mathrm{~L}$ powder, in which the temperature-dependent model parameters are readily extracted from the experimental measurement of surface and grain-boundary energies. The influences from the laser power and scan speed on key features, such as the porosity, surface morphology, temperature profile, geometry evolution as well as sintering stages of the grains and densification, are discussed respectively. It is hoped that the present work could enrich the modeling and computational toolkit which is practicable for the simulation of SLS-based additive manufacturing.

\section{RESULTS}

Non-isothermal phase-field formulation

A segment of a powder bed with the dimension of $250 \times 500 \times$ $100 \mathrm{\mu m}^{3}$ is considered in the simulation to reveal the microstructure evolution during SLS, as shown in Fig. 1a. Both conserved and non-conserved OPs $\rho$ and $\left\{\eta_{i}\right\}(i=1,2, \ldots, N)$ are employed to represent the powder bed with multiple particles. The conserved OP $\rho$ indicates the substance (unmelted and partially melted region), while the non-conserved OPs $\eta_{i}$ distinguish particles with different crystallographic orientations. ${ }^{38,43,46} \rho=1$ and $\rho=0$ represent the substance and the pore/atmosphere region, respectively. In each grain within the substance, only one of $\eta_{i}$ takes unity while others are zero (Fig. 1b). These grains and grain boundaries have $\rho=1$ (we assume the density variation across the grain boundary is negligible). When $\rho$ $=0$, no grain is present. This profile of OPs leads to the constraint $(1-\rho)+\sum \eta_{i}=1$. Their temporal evolution indicates the changes of $i$ the surface and grain boundaries, and thus represents the microstructure evolution during the SLS process.

Following the powder bed scenario, the entropy $\mathcal{S}$ of a finite subdomain $\Omega$ within the system can be written in a functional form as

$$
\mathcal{S}\left(e, \rho,\left\{\eta_{i}\right\}\right)=\int_{\Omega}\left[s\left(e, \rho,\left\{\eta_{i}\right\}\right)-\frac{1}{2} \kappa_{\rho}|\nabla \rho|^{2}-\frac{1}{2} \kappa_{\eta} \sum_{i}\left|\nabla \eta_{i}\right|^{2}\right] \mathrm{d} \Omega .
$$

Here, the local term of the entropy density $s$ is a function of the OPs $\rho,\left\{\eta_{i}\right\}$ and the internal energy density $e$. The positive constants $\kappa_{\rho}$ and $\kappa_{\eta}$ denote the contribution to the entropy density from the gradient of the order parameter according to the gradient thermodynamics. ${ }^{44,47}$ In order to relate this entropy to the free energy, which is also a functional of the OPs as well as temperature, we assume that the internal energy $\mathcal{E}$ is only the (a)

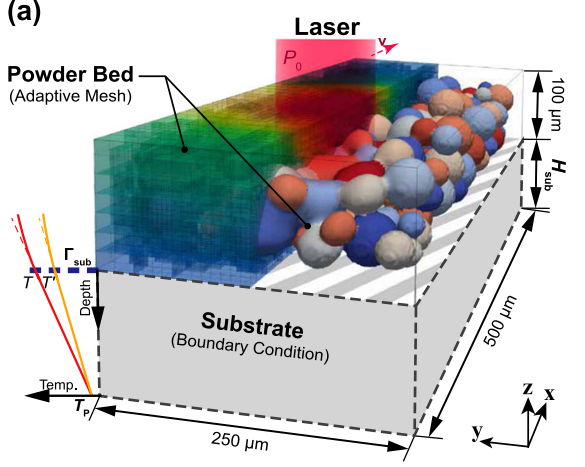

(b)

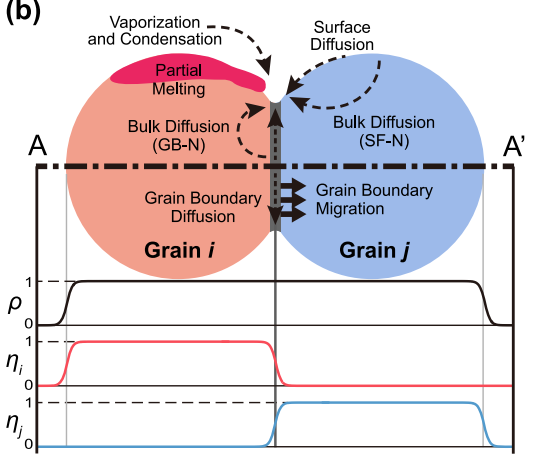

(c)

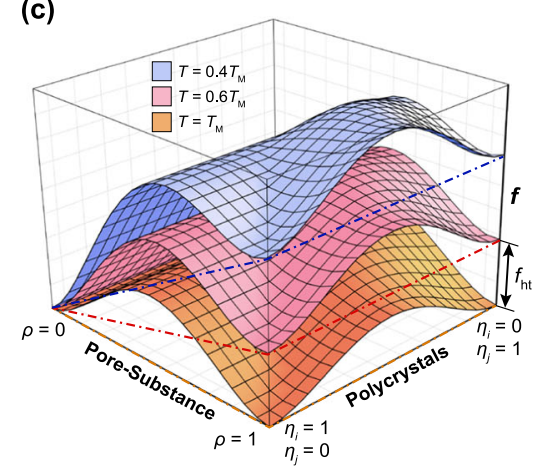

Fig. 1 a Schematics for the powder bed scenario of the SLS system with a thick substrate; $\mathbf{b}$ illustration of order parameters profile across $A-A^{\prime}$ section including physical phenomena, i.e., multiple mass transfer paths and grain boundary migration. Here the bulk diffusion 'GB-N' represents the path from grain boundary to neck through bulk, while 'SF- $N$ ' presents the path from surface to neck through bulk; c energy landscape of $f\left(T, \rho,\left\{\eta_{i}\right\}\right)$ at $T=0.4 T_{\mathrm{M}}, T=0.6 T_{\mathrm{M}}$ and $T=T_{\mathrm{M}}$

npj Computational Materials (2019) 81

Published in partnership with the Shanghai Institute of Ceramics of the Chinese Academy of Sciences 
integration of the internal energy density $e\left(T, \rho,\left\{\eta_{i}\right\}\right)$ through the subdomain. Then through Legendre transformation, ${ }^{48}$ the free energy $\mathcal{F}$ can be formulated as

$$
\begin{aligned}
\mathcal{F}(T, \rho, & \left.\left\{\eta_{i}\right\}\right)=\inf _{\mathcal{E}}\left[\mathcal{E}\left(T, \rho,\left\{\eta_{i}\right\}\right)-T \mathcal{S}\left(e, \rho,\left\{\eta_{i}\right\}\right)\right] \\
= & \int_{\Omega}\left[\inf _{e}\left[e\left(T, \rho,\left\{\eta_{i}\right\}\right)-T s\left(e, \rho,\left\{\eta_{i}\right\}\right)\right]\right. \\
& \left.+\frac{1}{2} T \kappa_{\rho}|\nabla \rho|^{2}+\frac{1}{2} T \kappa_{\eta} \sum_{i}\left|\nabla \eta_{i}\right|^{2}\right] \mathrm{d} \Omega .
\end{aligned}
$$

When the local entropy density $s\left(e, \rho,\left\{\eta_{i}\right\}\right)$ is lower bounded on $e$ $\left(T, \rho,\left\{\eta_{i}\right\}\right)$ at constant OPs, one can find a local free energy density $f$ $\left(T, \rho,\left\{\eta_{i}\right\}\right)$ which is also lower bounded on $T$ at constant OPs according to the Legendre transformation, i.e.

$$
f\left(T, \rho,\left\{\eta_{i}\right\}\right)=\inf _{e}\left[e\left(T, \rho,\left\{\eta_{i}\right\}\right)-T s\left(e, \rho,\left\{\eta_{i}\right\}\right)\right] \text {. }
$$

This relation leads to $d(f / T)=e d(1 / T) .{ }^{44}$ By integrating both sides of this relation w.r.t. $1 / T$, an explicit formulation of $f\left(T, \rho,\left\{\eta_{i}\right\}\right)$ can be obtained, which is

$$
f\left(T, \rho,\left\{\eta_{i}\right\}\right)=T \int e\left(T, \rho,\left\{\eta_{i}\right\}\right) \mathrm{d}\left(\frac{1}{T}\right) .
$$

It is further assumed that $e$ can be formulated as

$$
e\left(T, \rho,\left\{\eta_{i}\right\}\right)=e_{\mathrm{ht}}(T) \Phi_{\mathrm{ht}}\left(\rho,\left\{\eta_{i}\right\}\right)+e_{\mathrm{pt}}\left(\rho,\left\{\eta_{i}\right\}\right),
$$

where $e_{\mathrm{ht}}$ is the gain (or loss) of the internal energy density from the temperature change, and $e_{\mathrm{pt}}$ is the spatial distribution of the internal energy density w.r.t. OPs $\rho$ and $\left\{\eta_{i}\right\}$. The monotonic interpolating function $\Phi_{\text {ht }}\left(\rho,\left\{\eta_{i}\right\}\right)$ maps the heat to the regions with the certain value of the order parameters. We adopt the formulation $\Phi_{\mathrm{ht}}\left(\rho,\left\{\eta_{i}\right\}\right)=\xi\left(\underline{A} \rho+\underline{B} \sum_{i} \eta_{i}\right)$ with a fitting coefficient $\xi$. Integrating Eq. (4) by using Eq. (5), we obtain the following expression

$$
\begin{gathered}
f\left(T, \rho,\left\{\eta_{i}\right\}\right)=\Phi_{\mathrm{ht}}\left(\rho,\left\{\eta_{i}\right\}\right) T \int e_{\mathrm{ht}}(T) \mathrm{d}\left(\frac{1}{T}\right) \\
+e_{\mathrm{pt}}\left(\rho,\left\{\eta_{i}\right\}\right)-T s_{\mathrm{cf}}\left(\rho,\left\{\eta_{i}\right\}\right) .
\end{gathered}
$$

Here the term $s_{\mathrm{cf}}\left(\rho,\left\{\eta_{i}\right\}\right)$ is known as the configurational entropy since it has the same dimension as the entropy but only related to OPs. It can be verified that $s_{\mathrm{cf}}$ belongs to the entropy density $s(e, \rho$, $\left.\left\{\eta_{i}\right\}\right)$ by taking the inversion form of the Legendre transformation in Eq. (3), ${ }^{43,44}$ which yields

$$
\begin{gathered}
s\left(e, \rho,\left\{\eta_{i}\right\}\right)=\inf _{e}\left[\frac{e\left(T, \rho,\left\{\eta_{i}\right\}\right)}{T}-\frac{f\left(T, \rho,\left\{\eta_{i}\right\}\right)}{T}\right] \\
=\frac{1}{T} \int \operatorname{de}_{\mathrm{ht}}(T)+s_{\mathrm{cf}}\left(\rho,\left\{\eta_{i}\right\}\right),
\end{gathered}
$$

and the term $\int \mathrm{d} e_{\mathrm{ht}} / T$ meets exactly the definition of the thermal entropy. In order to find $f\left(T, \rho,\left\{\eta_{i}\right\}\right)$, terms $e_{\mathrm{ht}}(T), e_{\mathrm{pt}}\left(\rho,\left\{\eta_{i}\right\}\right)$ and $s_{\mathrm{cf}}(\rho$, $\left.\left\{\eta_{i}\right\}\right)$ should be properly formulated. Here $e_{h t}$ can be formulated as (note that we have set the internal energy of the atmosphere/ pores at melting point $T_{M}$ of the system as zero)

$$
e_{\mathrm{ht}}(T)=\left(c_{\mathrm{ss}}-c_{\mathrm{at}}\right)\left(T-T_{\mathrm{M}}\right)+\mathcal{L} \Phi_{\mathrm{M}}(\tau) \text {, }
$$

where $c$ is the volumetric specific heat, and the subscript 'ss' and 'at' denotes the substance and the atmosphere/pore, respectively. We denote the region with local temperature over or equal to the melting temperature $T_{\mathrm{M}}$ as the overheat region, and use interpolation function $\Phi_{M}(\tau)$ to indicate such region. It is expected that $\Phi_{\mathrm{M}}(\tau)$ takes one when $\tau=T / T_{\mathrm{M}} \rightarrow 1$ and takes zero when $\tau \rightarrow$ 0 . In such fashion, the latent heat for possible partial melting is simply represented by $\mathcal{L} \Phi_{M}(\tau)$.

On the other hand, $e_{\mathrm{pt}}$ and $s_{\mathrm{cf}}$ should consider the temperatureindependent potentials and configuration entropy among atmosphere/pores and grains in the substance mapped by OPs. In most cases, we only consider the jumps of both potential and configurational entropy on the surface and grain boundary, ${ }^{43,46,49}$ thus $e_{\mathrm{pt}}\left(\rho,\left\{\eta_{i}\right\}\right)$ and $s_{\mathrm{cf}}\left(\rho,\left\{\eta_{i}\right\}\right)$ can directly adopt the forms of
Landau-type polynomial proposed in refs. ${ }^{46,50}$, i.e.

$$
\begin{gathered}
e_{\mathrm{pt}}\left(\rho,\left\{\eta_{i}\right\}\right)=\underline{C}_{\mathrm{pt}}\left[\rho^{2}(1-\rho)^{2}\right]+ \\
\underline{D}_{\mathrm{pt}}\left[\rho^{2}+6(1-\rho) \sum_{i} \eta_{i}^{2}-4(2-\rho) \sum_{i} \eta_{i}^{3}+3\left(\sum_{i} \eta_{i}^{2}\right)^{2}\right], \\
s_{\mathrm{cf}}\left(\rho,\left\{\eta_{i}\right\}\right)=\underline{C}_{\mathrm{cf}}\left[\rho^{2}(1-\rho)^{2}\right]+ \\
\underline{D}_{\mathrm{cf}}\left[\rho^{2}+6(1-\rho) \sum_{i} \eta_{i}^{2}-4(2-\rho) \sum_{i} \eta_{i}^{3}+3\left(\sum_{i} \eta_{i}^{2}\right)^{2}\right] .
\end{gathered}
$$

Substituting Eqs. (8) and (9) into Eq. (6) yields

$$
\begin{gathered}
f\left(T, \rho,\left\{\eta_{i}\right\}\right)=\xi f_{\mathrm{ht}}(T)\left(\underline{A} \rho+\underline{B} \sum_{i} \eta_{i}\right)+\underline{C}\left[\rho^{2}(1-\rho)^{2}\right] \\
+\underline{D}\left[\rho^{2}+6(1-\rho) \sum_{i} \eta_{i}^{2}-4(2-\rho) \sum_{i} \eta_{i}^{3}+3\left(\sum_{i} \eta_{i}^{2}\right)^{2}\right],
\end{gathered}
$$

where

$$
\begin{gathered}
f_{\mathrm{ht}}(T)=\left(C_{\mathrm{ss}}-C_{\mathrm{at}}\right)\left[T \ln \frac{T}{T_{\mathrm{M}}}-\left(T-T_{\mathrm{M}}\right)\right]-\frac{T-T_{\mathrm{M}}}{T_{\mathrm{M}}} \mathcal{L} \Phi_{\mathrm{M}}(\tau), \\
\underline{C}=\underline{C}_{\mathrm{pt}}-\underline{C}_{\mathrm{cf}}\left(T-T_{\mathrm{M}}\right), \\
\underline{D}=\underline{D}_{\mathrm{pt}}-\underline{D}_{\mathrm{cf}}\left(T-T_{\mathrm{M}}\right) .
\end{gathered}
$$

Notice that Eq. (10) presents multiple minima including ( $\rho=0,\left\{\eta_{1}\right.$ $\left.\left.=0, \eta_{2}=0, \ldots, \eta_{N}=0\right\}\right)$ for an atmosphere/pores state and $(\rho=1$, $\left.\left\{\eta_{1}=1, \eta_{2}=0, \ldots, \eta_{N}=0\right\}\right),\left(\rho=1,\left\{\eta_{1}=0, \eta_{2}=1, \ldots, \eta_{N}=0\right\}\right),(\rho$ $\left.=1,\left\{\eta_{1}=0, \eta_{2}=0, \ldots, \eta_{N}=1\right\}\right)$ for grain states with different orientations. Model parameters $\underline{C}_{\mathrm{pt}}, \underline{D}_{\mathrm{pt}}, \underline{C}_{\mathrm{cf}}$, and $\underline{D}_{\mathrm{cf}}$ are related to the barrier heights between minima with the subscript 'pt' and ' $c f$ ' denote the contribution from the potential term or the configurational term, respectively. Heat term $f_{\text {ht }}$ tilts the "multi-well" due to the local heat variation, manifesting the variable thermodynamic stability due to the change of local thermal condition (Fig. $1 c)$. In this regard, the dimensionless model parameters $\underline{A}$ and $\underline{B}$ present the proportion of the heat contribution by different OPs while maintaining $\rho=0$ and $\rho=1$ as the equilibrium for mass conservation. Without introducing OPs to indicate the melting/ solidifying processes, the latent heat $\mathcal{L}$ would only influence the local thermodynamic stability along with the heat term. Those model parameters, as well as the gradient constants $\left(\kappa_{\rho}\right.$ and $\left.\kappa_{\eta}\right)$, are obtained from experimental measurements of the temperature-dependent surface and grain-boundary energies $\left(\gamma_{\mathrm{sf}}\right.$ and $\left.\gamma_{\mathrm{gb}}\right)$, and the grain boundary width. Coefficient $\xi$ is employed to favor the determination of model parameters by fitting the experimental results (see Supplementary Note 1).

The kinetics for conserved and non-conserved OPs, as well as $T$, are thereby derived based on obtained non-isothermal free energy density functional. Following assumptions are addressed for modeling the partial melting:

(1) Partial melting is locally restricted to individual particles when overheated, in which the melt flow is driven by the capillary pressure. This means the driven force of the melts can be described by variations of surface mean curvature and specific surface energy;

(2) Since partial melting is highly localized, it is negligible comparing the movement of the laser beam in length scale;

(3) Melts share the same material properties with the solid phase.

Detailed discussion and derivation are summarized in the Supplementary Note 2. As a result, kinetics of the conserved OP $\rho$ at the arbitrary space point $\mathbf{r}$ and time point $t$ can be formulated by the Cahn-Hilliard equation, i.e.

$\dot{\rho}(\mathbf{r}, t)=\nabla \cdot\left[M \nabla\left(\frac{\partial f}{\partial \rho}-T \kappa_{\rho} \nabla^{2} \rho\right)\right]$. 
Here the isotropic mobility $M$ is formulated to consider contributions not only from mass transfer paths through substance (ss), atmosphere (at), surface (sf), and grain boundary (gb), ${ }^{38,46,51,52}$ but also from the gain due to the partial melting (melt), i.e.

$$
\begin{aligned}
& M=\left[\Phi_{\mathrm{ss}} M_{\mathrm{ss}}^{\mathrm{eff}}+\Phi_{\mathrm{at}} M_{\mathrm{at}}^{\mathrm{eff}}+\Phi_{\mathrm{sf}} M_{\mathrm{sf}}^{\mathrm{eff}}+\Phi_{\mathrm{gb}} M_{\mathrm{gb}}^{\mathrm{eff}}\right]_{\mathrm{diff}} \\
& +\Phi_{\mathrm{M}}(\tau) M_{\text {melt }}^{\text {eff }} \text {, }
\end{aligned}
$$

where $\Phi_{\mathrm{ss}}, \Phi_{\mathrm{at}}, \Phi_{\mathrm{sf}}$, and $\Phi_{\mathrm{gb}}$ are also interpolating functions to indicate substance (including solid and liquid), atmosphere/pores, surface, and grain boundary, respectively, which obtain unity only in the corresponding region. They can be simply formulated as

$$
\begin{gathered}
\Phi_{\mathrm{ss}}=\rho^{3}\left(10-15 \rho+6 \rho^{2}\right), \quad \Phi_{\mathrm{at}}=1-\rho^{3}\left(10-15 \rho+6 \rho^{2}\right), \\
\Phi_{\mathrm{sf}}=16 \rho^{2}(1-\rho)^{2}, \quad \Phi_{\mathrm{gb}}=16 \sum_{i \neq j} \eta_{i}^{2} \eta_{j}^{2} .
\end{gathered}
$$

Notice that such formulation holds only when limited melting is assumed to occur around the surface of particles. In this regard, the contribution of the partial melting is treated more like an enhanced surface diffusion when $\tau \rightarrow 1$. Proper formulation where the melt flow is obtained by coupling with fluid dynamic equations (e.g. the Navier-Stokes equations) should be derived when melting dominates (a.k.a. the SLM process), and will be our future work.

On the other hand, evolution of $\left\{\eta_{i}\right\}$ is governed by the AllenCahn equation with the corresponding mobility $L$, i.e.

$\dot{\eta}_{i}(\mathbf{r}, t)=-L\left(\frac{\partial f}{\partial \eta_{i}}-T \kappa_{\eta} \nabla^{2} \eta_{i}\right)$.

The kinematic equation of temperature is formulated as

$$
\begin{gathered}
\frac{\partial e}{\partial T}[\dot{T}(\mathbf{r}, t)-\mathbf{v} \cdot \nabla T]+\frac{\partial e}{\partial \rho} \dot{\rho}(\mathbf{r}, t)+\sum_{i} \frac{\partial e}{\partial \eta_{i}} \dot{\eta}_{i}(\mathbf{r}, t) \\
=\nabla \cdot(k \nabla T)+q(\mathbf{r}),
\end{gathered}
$$

where the transient terms of OPs show the coupling with the microstructure evolution. The thermal conductivity $k$ adopts spatial distribution as $M$ in Eq. (12), i.e.

$k=\Phi_{\mathrm{ss}} k_{\mathrm{ss}}^{\mathrm{eff}}+\Phi_{\mathrm{at}} k_{\mathrm{at}}^{\mathrm{eff}}+\Phi_{\mathrm{sf}} k_{\mathrm{sf}}^{\mathrm{eff}}+\Phi_{\mathrm{gb}} k_{\mathrm{gb}}^{\mathrm{eff}}$.

The heat induced by the scanning laser is modeled as a source term following the surface Gaussian distribution moving with a velocity of $\mathbf{v}$. The heat source term $q(\mathbf{r})$ represents the volumetric energy deposition due to the radiative energy flux of the laser and can be formulated $\mathrm{as}^{6,7}$

$$
q[\mathbf{r}(x, y, z)]=\Phi_{s s} \beta P_{0} p_{x y}(x, y) p_{z}(a, \lambda, z),
$$

in which $P_{0}$ is the nominal laser power reaching the surface of the powder bed, $p_{x y}(x, y)$ is the surface Gaussian distribution. $p_{z}(a, \lambda$, $z$ ) is a penetration function, which takes the form proposed by Gusarov et al. $^{6}$. The hemispherical reflectivity $a$ indicates the influence of the powder material. The attenuation coefficient $\beta$ and the optical thickness $\lambda$ reflect the influence from the powder bed structure. The validation of Eq. (14) with $q(\mathbf{r})$ as in Eq. (16) is shown in Supplementary Fig. 2. For a loosely packed powder bed with particles of distributed diameters $d$, a thickness of $H_{\mathrm{pb}}$, and a porosity of $\varepsilon$, the effective value of these two parameters are calculated as

$$
\langle\beta\rangle=\frac{3}{2} \frac{1-\varepsilon}{\varepsilon}\left\langle\frac{1}{d}\right\rangle,\langle\lambda\rangle=\frac{3}{2} \frac{1-\varepsilon}{\varepsilon}\left\langle\frac{H_{\mathrm{pb}}}{d}\right\rangle .
$$

Notice that $d, H_{\mathrm{pb}}$ and $\varepsilon$ should change along with the microstructure evolution. Due to the difficulties to track all of them simultaneously, in this work we provided $\langle\beta\rangle$ and $\langle\lambda\rangle$ of the first step as non-temporal constants. On the substrate we set a heat conduction boundary condition to replace the mesh of the substrate (Fig. 1a), which has a similar fashion to the third-type BC of heat transfer problems, i.e.

$-(k \nabla T) \cdot \widehat{\mathbf{n}}=-\frac{k_{\text {sub }}}{H_{\text {sub }}}\left(\left.T\right|_{\Gamma_{\text {sub }}}-T_{\mathrm{P}}\right)$,

where $\widehat{\mathbf{n}}$ is the normal vector of the boundary $\Gamma_{\text {sub }} . H_{\text {sub }}$ and $k_{\text {sub }}$ are the thickness and the thermal conductivity constant of the homogeneous substrate, respectively. The bottom of the substrate is set with a fixed temperature $T_{\mathrm{P}}$ as the pre-heating temperature.

Optimized assignment of the order parameters for grain orientation

Generally, the number of non-conserved OPs $\left\{\eta_{i}\right\}$ is required to be the same as the number of the grains to statistically retain the uniqueness of each crystalline orientation. ${ }^{53,54}$ However, such computation is expensive due to a large amount of degree of freedoms (DOFs), and is inefficient since only a few variables are nonzero at one point in the domain. To solve those problems, methods that can drastically reduce the computational cost while retaining the uniqueness of the grains have been proposed in the recent decade. ${ }^{55-57}$ The basic principle of such methods is to assign the same $\eta_{i}$ to grains which are sufficiently spaced, and remap all $\left\{\eta_{i}\right\}$ when certain grains with an identical $\eta_{i}$ tend to coalesce. This idea makes it possible to use less amount of DOFs to simulate the evolution of the polycrystalline structure. Using the grain tracking algorithm proposed by Permann et al. in, ${ }^{57}$ it is able to simulate more than 1000 grains with a minimum of eight $\eta_{i}$ in $2 \mathrm{D}$ and 28 in 3D in the grain growth problems. Due to the on-site heating and rapid cooling during SLS, global and long-term grain coalescence is absent, in contrast to the grain growth process. So the profile of non-conserved OPs will not change violently during SLS, and the optimized assignment of OPs could further reduce the computation cost.

In this work, we translate the problem of OPs' assignment into a classical minimum coloring problem (MCP). By solving the MCP with the Welsh-Powell algorithm, ${ }^{58}$ we obtain the optimized profile with a minimum number of $\left\{\eta_{i}\right\}$. The workflow of the process is presented in Supplementary Fig. 1. In the beginning, the adjacency matrix of the powder bed, demonstrating the "adjacent neighbors" of each powder, is generated according to the criterion of adjacency (Supplementary Fig. 1b). If two particles/grains which are spaced less than this critical distance, they can be considered as "adjacent neighbors". Here the distance between the largest powder and its next-nearest neighbor in hexagonal close packing is considered as the criterion of adjacency. Then, the map $G(\mathbb{V}, \mathbb{E})$ is established based on the adjacency matrix, which consists of the unique particle $v_{i}(\mathbf{r}, R)$ as the vertices $\mathbb{V}\left\{\nu_{i}\right\}$ and corresponding degree of adjacency $\mathbb{E}\left\{\epsilon_{i}\right\}$, i.e., there are $\varepsilon_{i}$ particles being adjacent to the one indexed as $v_{i}$ (Supplementary Fig. 1c). Once the map is established, the algorithm will iteratively assign the available $\eta_{i}$ to non-adjacent and non-assigned particles, until all of them have been assigned with $\left\{\eta_{i}\right\}$. The particles indices remain identical during this iterative assignment, and can be later translated by the grain tracking algorithm. In this work, for the powder bed with particle diameters ranging from 20 to $50 \mu \mathrm{m}$, eight $\left\{\eta_{i}\right\}$ are sufficient to represent about 200 particles/grains (Supplementary Fig. 1d, 1e). For another simulation attempt using a loosely packed powder bed with about 400 uniformly sized particles, only six $\left\{\eta_{i}\right\}$ are sufficient. In this way, the number of DOFs is remarkably reduced.

\section{SLS single scan simulations}

Here we present the results from the SLS single scan simulations informed by measured properties of the type $316 \mathrm{~L}$ stainless steel (SS316L) in the argon atmosphere. SS316L belongs to the family of austenite stainless steel. For such type of stainless steel, no solid structural transition is expected. ${ }^{59}$ Assuming the isotropic diffusion and grain boundary migration, the mobilities $L$ and $M$ are adopted 
from the self-diffusivity $D_{\text {path }}^{\text {eff }}$ through possible paths (path $=s s$, at, $\mathrm{sf}$, and $\mathrm{gb}$ ) and grain boundary mobility $G_{\mathrm{gb}}^{\text {eff }} 40,43,60$ i.e.

$M_{\text {path }}^{\text {eff }}=\frac{D_{\text {path }}^{\text {eff }}}{2(\underline{C}+\underline{D})}, L^{\text {eff }}=\frac{G_{\mathrm{gb}}^{\text {eff }} \gamma_{\mathrm{gb}}}{T \kappa_{\eta}}$.

Details of the material-related quantities are listed in Supplementary Note 3, and the procedure to obtain model parameters $\left(\underline{A}, \underline{B}, \underline{C}_{\mathrm{pt}}, \underline{C}_{\mathrm{cf}}, \underline{D}_{\mathrm{pt}}, \underline{D}_{\mathrm{cf}}, \kappa_{\eta}\right.$ and $\left.\kappa_{\rho}\right)$ are elaborated in Supplementary Note 1 . The reference length scale is set as $\bar{l}=1 \mu \mathrm{m}$, and the time scale as $\bar{t}=1 \mu \mathrm{s}$. The thickness of the powder bed layer is $H_{\mathrm{pb}}=$ $65 \mu \mathrm{m}$, and of the substrate is $H_{\text {sub }}=1000 \mu \mathrm{m}$. The powder bed consists of particles with distributed diameters $d$ ranging from 20 to $50 \mu \mathrm{m}$. The distribution of $d$ is shown in Supplementary Fig. 3a. According to Eq. (17), the effective attenuation coefficient and optical thickness of the powder bed are calculated as $\langle\beta\rangle=0.089$ and $\langle\lambda\rangle=5.8$. The Gaussian distribution of the laser beam $p_{x y}(x, y)$ has the full width at half maximum FWHM. Note that the surface integral of $p_{x y}(x, y)$ reaches $75.8 \%$ of $P_{0}$ in the region with a diameter of FWHM, which we defined as the half-maximum (HM) region, and reaches $99.5 \%$ in the region with a diameter of $2 \times$ FWHM. So we regard the region $2 \times$ FWHM as the nominal beam spot. And scan speed $v$ is simply defined as $v=|\mathbf{v}|$. The melting point of SS316L is read as $T_{M}=1700 \mathrm{~K}$. Temperature of the powder bed is initialized as $\left.T\right|_{t=0}=0.4 T_{\mathrm{M}}=680 \mathrm{~K}$, which is also the value of pre-heating temperature $T_{\mathrm{P}}$.

Figure $2 \mathrm{a}$ presents the simulated microstructure evolution under the single scan with $\mathrm{FWHM}=100 \mu \mathrm{m}, P_{0}=20 \mathrm{~W}$ and $v=100 \mathrm{~mm} / \mathrm{s}$. The SLS induced physical phenomena around the laser beam spot are shown in Fig. 2b. Due to the laser scan, temperature of the powder bed obviously increases, as shown in Fig. 2a. Overheat region where $T \geq T_{M}$ is noted with continuous colormap. Only within such region, particles are able to be partially melted. Reduction of the total surface energy (or surface capillary pressure) makes the melts flow from the convex to the concave. This kind of melt flow results in a continuous piece with coarsened grains once cooled down. In the region with a maximum temperature lower than $T_{\mathrm{M}}$, no partial melting occurs. But the temperature is still high enough to activate diffusion and thus induce necking among adjacent particles. From the isotherms in Fig. 2a, we can estimate the local temperature gradient around the partial melting region and the pore region as high as $50 \mathrm{~K} / \mu \mathrm{m}$ and $100 \mathrm{~K} / \mu \mathrm{m}$, respectively. Such large-gradient temperature fields may induce the mass transfer due to temperature-induced inhomogeneity of the surface energy. This also distinguishes SLS from conventional isothermal sintering. ${ }^{43}$ In addition, we compare the simulated surface morphologies along with the experimental observations in ref. ${ }^{26}$ on a powder bed
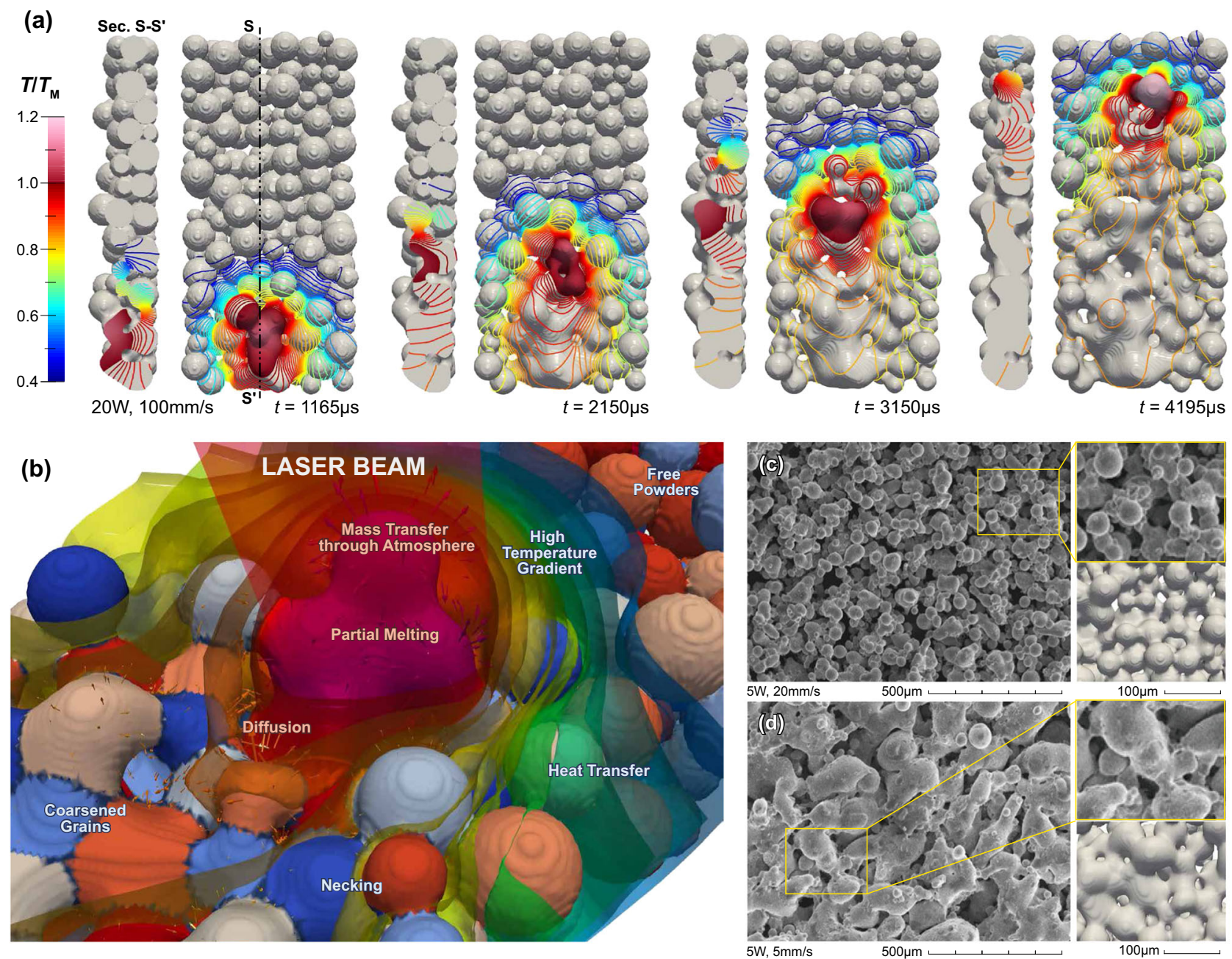

Fig. 2 a Simulation results on SLS processing of SS316L powder bed with $P_{0}=20 \mathrm{~W}$ and $v=100 \mathrm{~mm} / \mathrm{s}$. Overheat region where $T \geq T_{\mathrm{M}}$ is noted with continuous colormap while one with $T<T_{M}$ is noted with isotherms; $\mathbf{b}$ phenomena and characteristics around the laser beam spot during the process. Comparison of the surface morphology between the simulated and experimental results in the case of $\mathrm{c} P_{0}=5 \mathrm{~W}, v=20 \mathrm{~mm} / \mathrm{s}$ and $\mathbf{d} P_{0}=5 \mathrm{~W}, v=5 \mathrm{~mm} / \mathrm{s}$. SEM images are reprinted with permission from Liu et al. ${ }^{26}$ 
with Fe powders of $50 \mu \mathrm{m}$ in diameter and SS316 $\mathrm{L}$ matrix under a constant $P_{0}=5 \mathrm{~W}$ for $v=20 \mathrm{~mm} / \mathrm{s}$ and $v=5 \mathrm{~mm} / \mathrm{s}$ as presented, respectively. As for $v=20 \mathrm{~mm} / \mathrm{s}$ in Fig. 2c, powder bed shows a poor binding and the necking among adjacent particles is very weak. As for $v=5 \mathrm{~mm} / \mathrm{s}$ in Fig. $2 \mathrm{~d}$, on the other hand, continuous pieces are formed after the SLS processing, indicating better binding of particles.

Laser power and scan speed are important processing parameters for SLS, through which one can effectively control the properties (e.g. porosity and grain size) of the processed components. Here we investigate the influence of scan speed and laser power by performing a series of simulations. As shown in Fig. 3 , the morphology and porosity of the SLS processed powder bed are presented with different laser power and scan speed. If without further explanation, we set $\mathrm{FWHM}=100 \mu \mathrm{m}$ for all following simulations. The porosity map can be roughly divided into two regions by the dash-dotted line as shown in Fig. 3a. The lower-right region shows less bound particles, while the upper-left region shows more continuous pieces. When decreasing the scan speed while fixing the laser power, more particles are bound to create more continuous pieces, i.e., less porosity will be achieved in the final components (comparing Fig. $3 b_{4}-3 b_{7} ; 3 b_{8}-3 b_{10}$; and $\left.3 b_{3}, 3 b_{12}-3 b_{11}\right)$. It is similar for the case when scan speed is fixed and the laser power is increased (comparing Fig. $3 b_{1}-3 b_{4} ; 3 b_{5}$, $3 b_{8}, 3 b_{12}$; and $\left.3 b_{6}, 3 b_{10}-3 b_{11}\right)$.

\section{DISCUSSION}

In this section, we explicitly discuss the influences from laser power and scan speed on features such as the temperature profile, geometry, and corresponding sintering stages of particles/ grains, and microstructure densification. Temperature profile in SLS is directly related to the processing parameters. It is an important indicator to determine whether partial melting could occur during SLS. In our work here, transient temperature profile interacts with microstructure evolution. Explicitly, heat transfer receives influences from microstructure evolution through transient terms of OPs as shown in Eq. (14). Kinetics of OPs simultaneously receive influences from local temperature variation through temperature-dependent local and gradient term as well, shown in Eqs. (11) and (13). Therefore, we can simulate the temperature profile not only on the particle scale, but also in a way close to the realistic setup.

Figure 4a presents the top view and the section view of the temperature profile at the point when the laser beam has passed $350 \mu \mathrm{m}$ on the powder bed with different processing parameters. The half-maximum (HM) range and the nominal beam spot of the
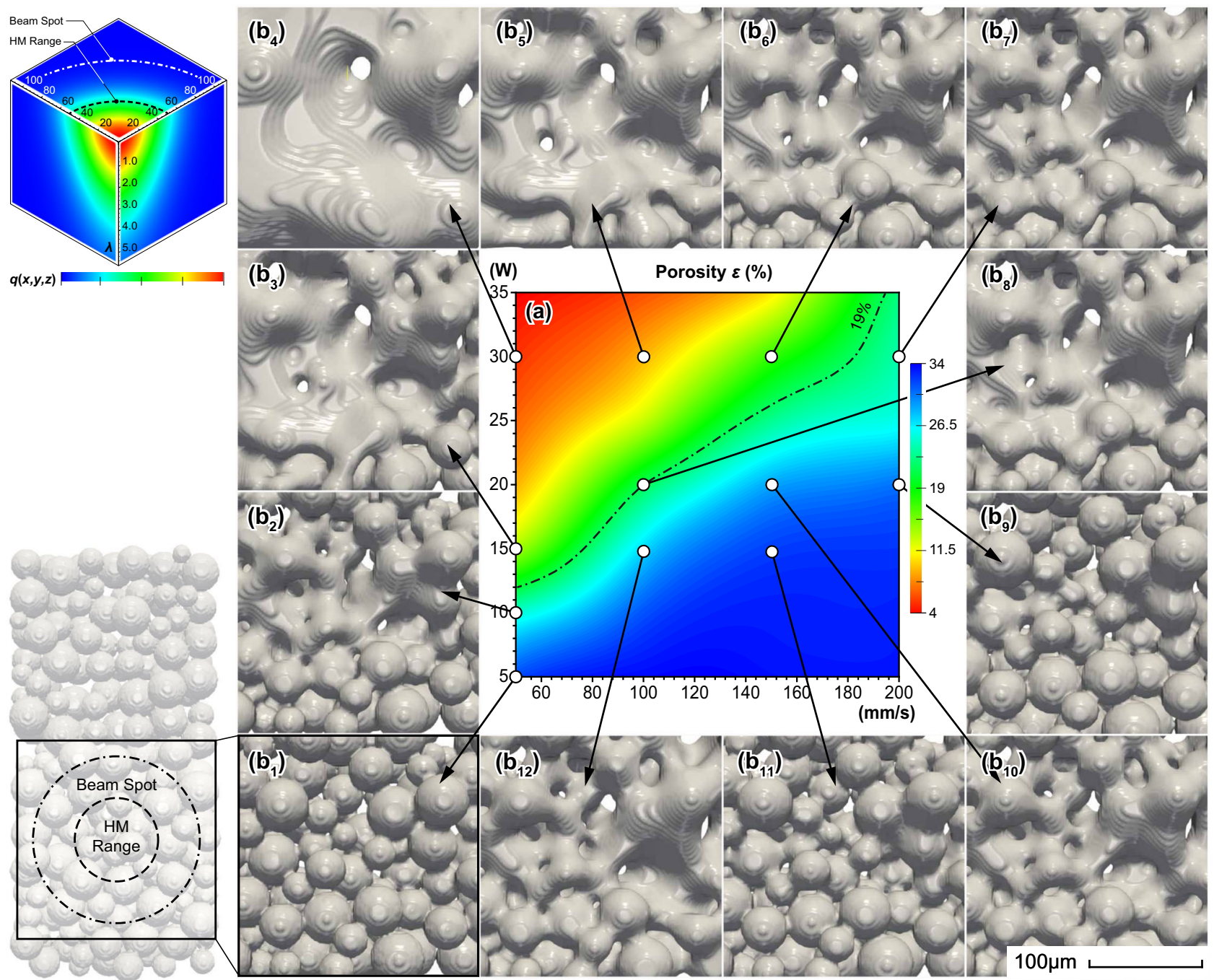

Fig. 3 a Porosity map and $\mathbf{b}_{1}-\mathbf{b}_{12}$ morphology of the SLS processed powder bed using different laser power and scan speed. The dash-dotted line in $\mathbf{a}$ is the isoline of $19 \%$ porosity, i.e., the initial porosity is reduced by half. Left inset: schematics of the presenting segment, the HM range (dashed line) as well as the beam spot (dash-dotted line), and the distribution of the volumetric energy deposition 


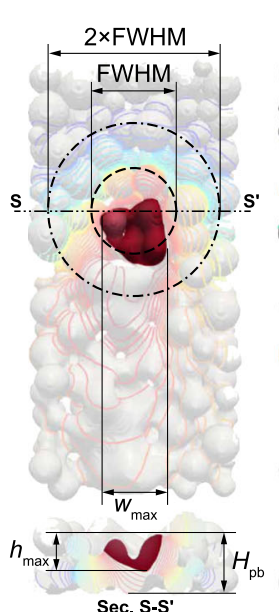

(a)
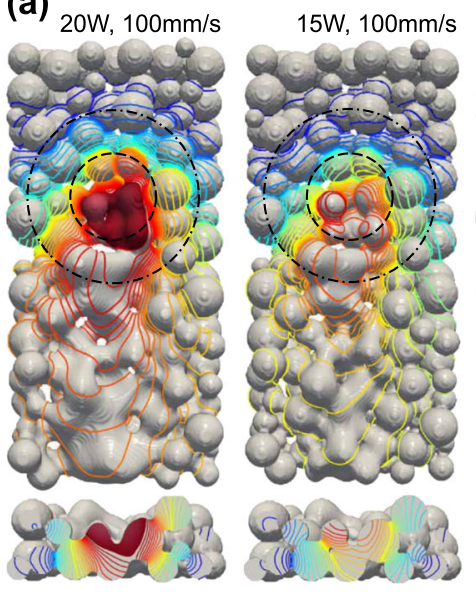

(b)

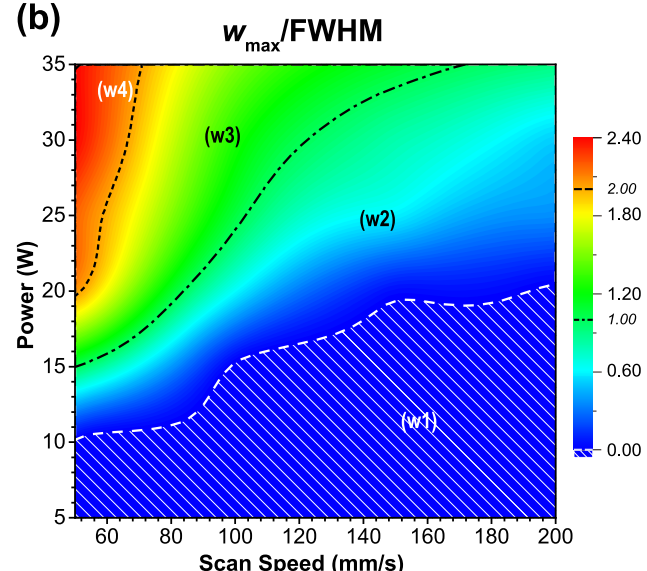

$30 \mathrm{~W}, 100 \mathrm{~mm} / \mathrm{s}$
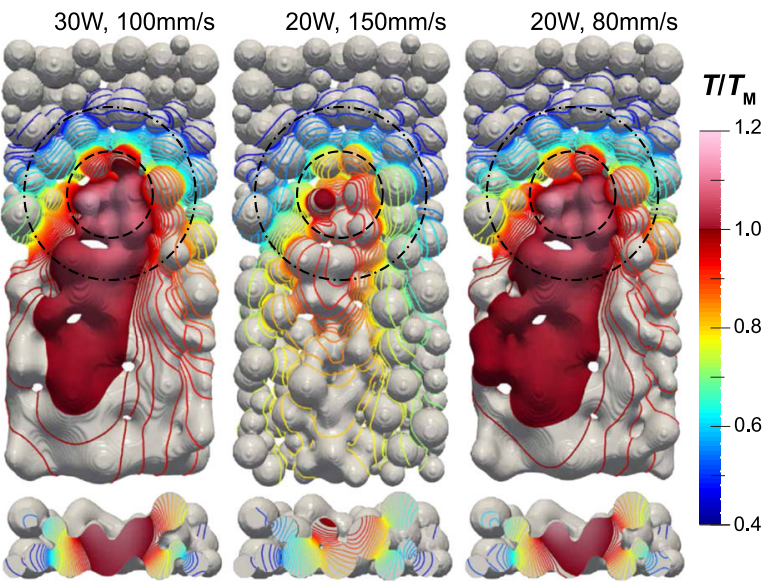

(c)

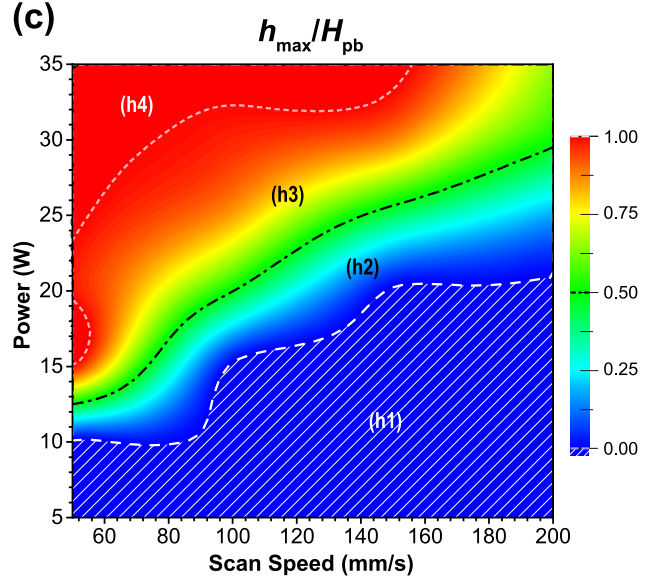

Fig. 4 a Temperature profile at the point when the laser beam has passed $350 \mu \mathrm{m}$ on the powder bed with different processing parameters. Overheat region with $T \geq T_{M}$ is noted with continuous colormap while one with $T<T_{M}$ is noted with isotherms. Left inset: schematics of the presenting section (Sec. S-S'), the HM range (dashed line) as well as the beam spot (dash-dotted line), and the geometry of the overheat region which is characterized by width $w$ and depth $h$. Maps of $\mathbf{b}$ the normalized width and $\mathbf{c}$ the normalized depth of the overheat region along with laser power and scan speed

laser are also indicated in the figure. It generally presents an inhomogeneous temperature field on the powder bed. In the front of the moving beam spot, isotherms are very dense and become sparse once the beam spot has passed away, indicating rapid heating and a relatively slow cooling. In this regard, increasing the laser power and decreasing the scan speed can both improve the heat accumulation around the beam spot. As we mentioned that partial melting is only possible within the overheat region where $T \geq T_{\mathrm{M}}$ (noted with continuous colormap), we can only see some particles are partially melted in the case of $P_{0}=20 \mathrm{~W}$ and $v=$ $100 \mathrm{~mm} / \mathrm{s}$. When increasing the laser power (e.g. $P_{0}=30 \mathrm{~W}$ and $v$ $=100 \mathrm{~mm} / \mathrm{s}$ in Fig. 4 a) or decreasing the scan speed (e.g. $P_{0}=$ $20 \mathrm{~W}$ and $v=80 \mathrm{~mm} / \mathrm{s}$ in Fig. $4 \mathrm{a}$ ), the overheat region becomes more noticeable. In these cases, considerable melts would be generated by partial melting, turning the mechanism of liquidstate sintering to be dominant. On the other hand, decreasing the laser power (e.g. $P_{0}=15 \mathrm{~W}$ and $v=100 \mathrm{~mm} / \mathrm{s}$ in Fig. 4a) or increasing the scan speed (e.g. $P_{0}=20 \mathrm{~W}$ and $v=150 \mathrm{~mm} / \mathrm{s}$ in Fig. 4a) can effectively reduce the partial melting, and in these cases the mechanism of solid-state sintering is dominant.

To systematically analyze the influence of processing parameters on the temperature profile and classify the possible dominant mechanism involved in SLS processing, we map the width (normalized with FWHM) and the depth (normalized with $H_{\mathrm{pb}}$ ) of the overheat region (left inset of Fig. 4a) along with the processing parameters, as shown in Fig. $4 b$, c. To help the discussion, each map is divided into four areas, and the processing parameters inside a certain area are corresponding to similar microstructure features. For the normalized overheat width map (Fig. 4b) we have: (w1) there is no overheat region; (w2) overheat region is extremely localized within the HM range (diameter of FWHM); (w3) overheat region is wider than the HM range, yet still localized within the beam spot (diameter of $2 \times$ FWHM); (w4) overheat region is wider than beam spot. For the normalized melting depth map (Fig. 4c) we have: (h1) there is no overheat region; (h2) overheat region is localized within half of the powder bed thickness; (h3) overheat region exceeds half of the powder bed thickness; and (h4) overheat region equals (or probably exceeds) the powder bed thickness. To sum up, there is only dominant solid-state sintering in the overlapped area of (w1) and (h1), thus no contribution from partial melting need to be considered. In the areas (w2), (w3), and (h2), and (h3), particles coexist with melts. Therefore, it is necessary to consider the partial melting in such regions. However, in the overlapped area of (w2) and (h2), the partial melting is restricted to the individual particle and then quickly vanished after cooling down. This process is closer to solid-state sintering. In other areas, there are more melts mixed with the unmelted particles, leading to typical liquid-state sintering. Finally, the dominant melting and solidification occur in regions (w4) and (h4) (a.k.a. the SLM process). In such regions the partial melting assumptions no longer hold, and the proper model coupled with fluid dynamics of the melt is desired. 
Due to the inhomogeneous temperature profile, particles/grains during SLS may undergo different binding mechanisms depending on local thermodynamic conditions. Some particles around the beam spot may suffer from the partial melting. Others may be sintered together since the temperature is still sufficiently high to activate the diffusion and the grain boundary migration. Inhomogeneity of local temperature distribution would cause considerable differences in both diffusivity and grain boundary mobility, which follow the Arrhenius equation, between the hottest and coolest ends of the powder bed. For instance, the surface diffusivity at the hottest end of a partially melted particle is about 100 times larger than that on at the coolest end in this work. As a result, grains in different sintering stages (majorly judged by grain geometry ${ }^{61}$ ) could be simultaneously observed, in contrast to that during the conventional sintering where the uniform temperature is firmly controlled. Those grains in different sintering stages may significantly affect the densification (or porosity) of the final product. With the help of grain tracking algorithm, we can track the particle/grain located at any position in the powder bed and investigate the sintering stages of certain grain during the process.

Figure 5 a presents the geometry evolution of particles/grains of the same processing parameters of $P_{0}=20 \mathrm{~W}$ and $v=100 \mathrm{~mm} / \mathrm{s}$. Here the volume and the average temperature of the $i$-th particle/ grain are calculated as

$V=\int \rho \delta\left(\Omega_{i}\right) \mathrm{d} \Omega, T_{\text {avg }}=\frac{\int \rho T \delta\left(\Omega_{i}\right) \mathrm{d} \Omega}{\int \rho \delta\left(\Omega_{i}\right) \mathrm{d} \Omega}$,

where $\delta\left(\Omega_{i}\right)$ is the delta function which takes one inside the domain $\Omega_{i}$ of the $i$-th particle/grain. $\Omega$ represents the simulation domain. The volume variation $\Delta V$ is thereby calculated as the absolute difference between the current volume $V$ and the initial volume $V_{0}$. When the laser beam passes, the average temperatures rise to the peak, then gradually drop. The volume variation, however, starts to rise when the average temperature reaches $0.6 T_{\mathrm{M}}$. After reaching the maximum $T_{\text {avg, }}$ the rate of volume variation gradually drops, and the grain volume tends to be constant. It can be seen that grains A, D, and E (Fig. 5a) with the same $y$ coordinate present similar polyhedral shapes with arched surfaces $\left(g_{A}, g_{D}\right.$, and $\left.g_{E}\right)$ at the end of the scan $(5000 \mu s)$, showing a resemblance to the grains during the intermediate stage of the sintering. Grain $\mathrm{F}$ is exactly located beneath the partial melting region. It also turns into a polyhedral shape $\left(g_{F}\right)$ of the grains during the intermediate stage. On the other hand, grains $A, B$, and $C$ with the same $z$ coordinate present different stages. Among these three grains, grain $B$ is located farthest from the beam center and eventually turns into a shape which is less polyhedral, but similar to the one formed by pure necking without subsequent packing $\left(g_{B}\right)$, which is the symbolic feature of the grains during the starting stage of sintering. Grain $C$ is located closest to beam center, whose $T_{\text {avg }}$ firstly rises above $T_{\mathrm{M}}$ then quickly drops. It eventually turns into a grain $\left(\mathrm{g}_{\mathrm{C}}\right)$ with a smooth and relatively wider top and polyhedral bottom. Partial melting may be the reason for such smooth wide top, where grain $C$ gets overheated and hence gain extra mobility $\Phi_{\mathrm{M}}(\tau) M_{\text {melt }}^{\text {eff }}$ for the mass transfer shown in Eq. (12). $T_{\text {avg }}$ of grain $C$ being shortly over $T_{M}$ also confirm the existence of the partial melting. Apart from this, features like the polyhedral bottom can be found from the highly packed grains during the final stage of the sintering.

Likewise, we present the geometry evolution of the same particle/grain (grain A) with various processing parameters in Fig. $5 \mathrm{~b}$. It can be seen that increasing the laser power and decreasing the scan speed can eventually accelerate the sintering of the grain. For $v=100 \mathrm{~mm} / \mathrm{s}$, the grain is polyhedral as the intermediate stage at $P_{0}=20 \mathrm{~W}\left(\mathrm{~g}_{3}\right)$, but is round with a top-surface necking as the starting stage at $P_{0}=10 \mathrm{~W}\left(\mathrm{~g}_{5}\right)$. For the cases of $P_{0}$ $=30 \mathrm{~W}, v=100 \mathrm{~mm} / \mathrm{s}$ and $P_{0}=20 \mathrm{~W}, v=80 \mathrm{~mm} / \mathrm{s}$, the grain with a smooth wide top and a polyhedral bottom is obtained as the final stage $\left(g_{1}\right.$ and $\left.g_{2}\right)$.

As the sintering progresses, grains get coarser and more packed, meanwhile the pores shrink or even vanish, leading to the nominal volume shrinkage and porosity reduction of the processed components. Such phenomena are collectively known as the densification. In ref. ${ }^{19}$ Simchi proposed that transient porosity during densification of laser sintered metal follows the first-order kinetics law as

$\dot{\varepsilon}=-\kappa \varepsilon$,

where $K$ is defined as the sintering rate constant. In Fig. 6a we present the temporal evolution of $\ln \left(\varepsilon / \varepsilon_{0}\right)$ of the three selected segments and the whole powder bed with an initial porosity $\varepsilon_{0}$ under the processing parameters of $P_{0}=20 \mathrm{~W}$ and $v=100 \mathrm{~mm} / \mathrm{s}$. The average temperature $T_{\text {avg }}$ of each segment is also calculated according to Eq. (20). These three segments are with the same geometry (length $100 \mu \mathrm{m}$, width $250 \mu \mathrm{m}$ ), but located at different positions in the powder bed. It can be seen that when the laser beam scans into a certain segment, $T_{\text {avg }}$ rises and $\ln \left(\varepsilon / \varepsilon_{0}\right)$ is almost linearly decreased there. Once the laser beam is about to leave the certain segment, the $T_{\text {avg }}$ reaches its maximum then starts to drop, while $\ln \left(\varepsilon / \varepsilon_{0}\right)$ is slowly decreased. In contrast, In $\left(\varepsilon / \varepsilon_{0}\right)$ of the whole powder bed presents an approximately linear trend, with a fitted slope as $k=7 \times 10^{-5} \mathrm{~s}^{-1}$, as shown in Fig. 6a. This demonstrates that Eq. (21) is valid in this model to describe the porosity evolution of the powder bed combining all contributions from each finite segments, which is also in agreement with the experimental measurement in ref. ${ }^{19}$. According to Fig. $6 \mathrm{~b}$, we can see the final microstructure $(t=5000 \mu \mathrm{s})$ around the segment center shows the resemblance to the microstructure during the intermediate stage of sintering. ${ }^{61}$ In this stage, grains have already been packed together while most of the open pores have been eliminated or rearranged into a roundly closed shape. Whereas the microstructure around the segment margin presents less packed but more necked grains surrounded by tunnel-like open pores, manifesting the characteristics during the initial stage of sintering. ${ }^{61}$

In Fig. $6 c$ we present the temporal evolution of $\ln \left(\varepsilon / \varepsilon_{0}\right)$ under different processing parameters. Segment II is selected to present the influence of processing parameters on temporal $T_{\text {avg, }}$ which shows a similar trend as in Fig. 6a. The approximately linear trend of $\ln \left(\varepsilon / \varepsilon_{0}\right)$ is still obvious. Both increasing the laser power and decreasing the scan speed result in the increase of the maximum $T_{\text {avg }}$ and the rate constant $\kappa$. Final microstructures of segment II under various processing parameters are shown in Fig. $6 \mathrm{~d}$. We set the microstructure of segment II of $P_{0}=20 \mathrm{~W}$ and $v=100 \mathrm{~mm} / \mathrm{s}$ as the reference and compare it with cases with other processing parameters. For the cases with fixed $v=100 \mathrm{~mm} / \mathrm{s}$ and $P_{0}=10,15$, and $20 \mathrm{~W}$, densification rarely occurs and only necking with tunnel-like open pores appears, also manifesting the characteristics of the initial stage of sintering. For the case of $P_{0}=30 \mathrm{~W}$ and $v=100 \mathrm{~mm} / \mathrm{s}$, however, the microstructure is significantly densified with packed grains and almost no pores, showing the characteristics of the final stage of the sintering where the grain coarsening dominates. For the case of $P_{0}=20 \mathrm{~W}$ and $v=80 \mathrm{~mm} / \mathrm{s}$, the microstructure densification occurs, but there are still some round pores near the segment margin, indicating the transition from the intermediate stage to the final stage of sintering.

Here we further discuss the relation between the processing parameters and the sintering rate as well as the final densification. We define the specific energy input $\Psi$ and densification factor $\varrho$ as

$\Psi=\frac{P}{h w v}, \varrho=\frac{\varepsilon_{0}-\varepsilon}{\varepsilon_{0}-\varepsilon_{\min }}$

In Eq. (22), $P$ and $v$ are the laser power and the scan speed of the laser beam, respectively. $h$ and $w$ are the thickness and width of the scan track, respectively. $\varepsilon_{\min }$ is the minimum attainable 
(a)

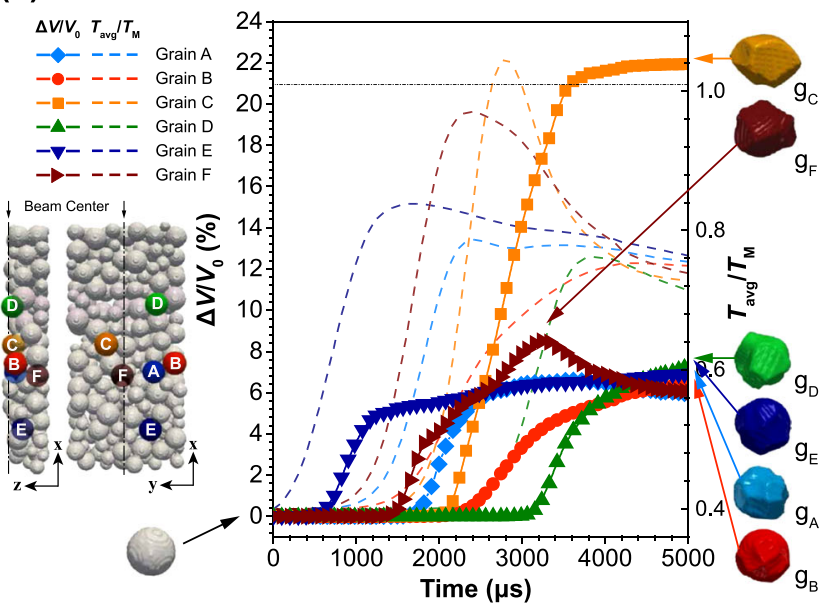

(b)

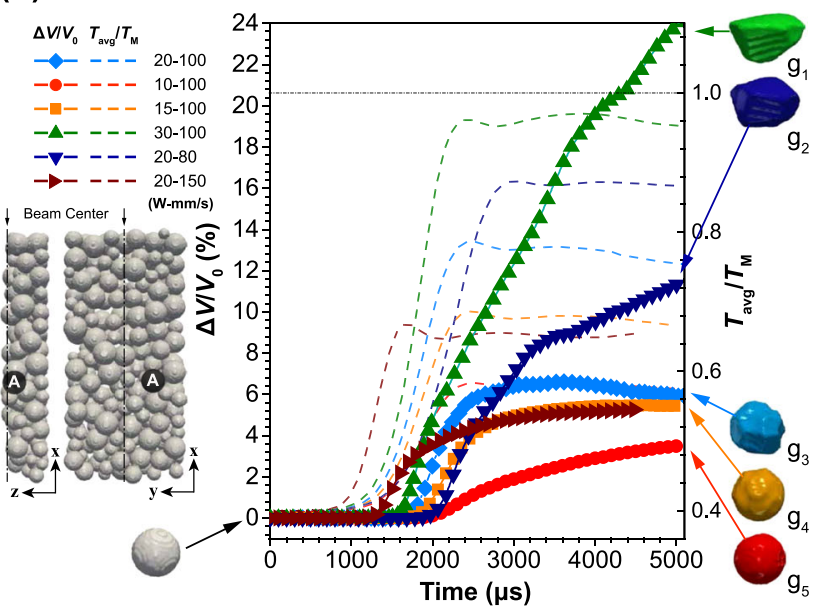

Fig. 5 a The normalized size variation of the particles/grains located at positions A-F as shown in left inset under the processing parameters of $P_{0}=20 \mathrm{~W}, v=100 \mathrm{~mm} / \mathrm{s}$. Final geometries of corresponding particles/grains are presented in insets $\mathrm{g}_{\mathrm{A}}-\mathrm{g}_{\mathrm{F}}$; $\mathbf{b}$ the normalized size variation of the particles/grains located at position A as shown in left inset under various processing parameters. Final geometries of selected particles/ grains are presented in $\mathrm{g}_{1}-\mathrm{g}_{5}$

(a)

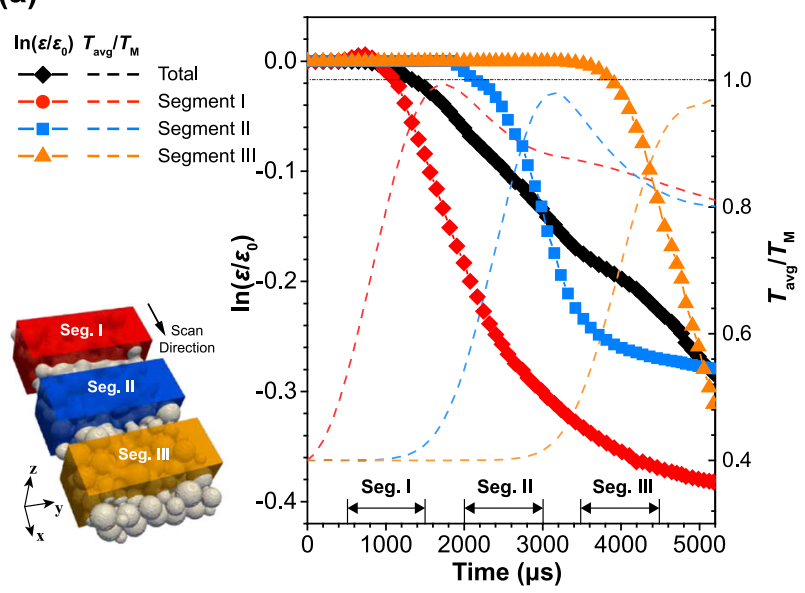

(b)
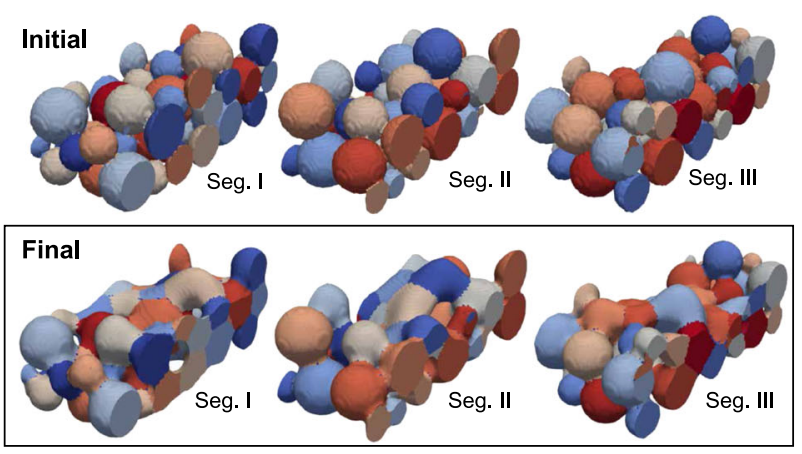

(c)

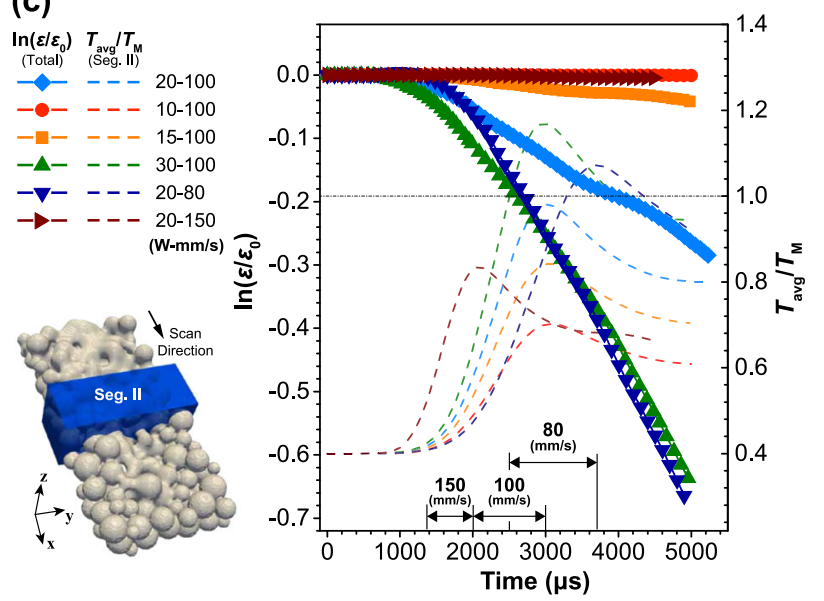

(d)

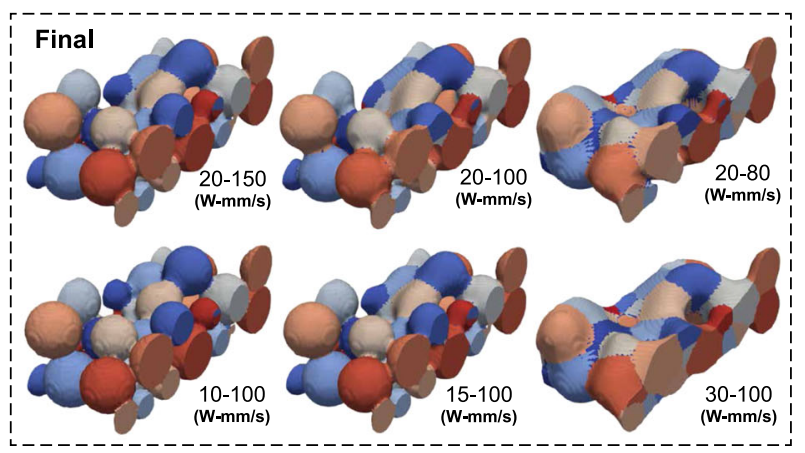

Fig. 6 a Temporal evolution of $\ln \left(\varepsilon / \varepsilon_{0}\right)$ and the average temperature $T_{\text {avg }}$ of the different segments and the whole powder bed under the processing parameters of $P_{0}=20 \mathrm{~W}, v=100 \mathrm{~mm} / \mathrm{s}$, and $\mathbf{b}$ the final microstructures of selected segments; $\mathbf{c}$ temporal evolution of In $\left(\varepsilon / \varepsilon_{0}\right)$ of the whole powder bed and the $T_{\text {avg }}$ of the same segment of the powder bed under the various processing parameters, and $\mathbf{d}$ the final microstructures of the segment. Time windows shown in $\mathbf{a}, \mathbf{c}$ indicate the periods when the beam scans on the corresponding segment

porosity in a sintered part, which ranges between 0.02 and 0.3 depending on the material properties. To avoid the deviation due to the less densified margin of the powder bed, we choose a $\mathrm{FWHM}=100 \mu \mathrm{m}$ of the power density of the laser as the track width $w$, and $P=75.8 \% P_{0}$ (the surface integral of the power density reaches $75.8 \%$ of $\left.P_{0}\right)$. $h$ takes $H_{\mathrm{pb}}=65 \mu \mathrm{m}$ and $\varepsilon_{\min }=3 \%$. As shown in Fig. $7 a$, the relation $\kappa$ vs $\Psi$ can be linearly fitted with a slope of $9.51 \times 10^{-3}$. Similarly, linear fitting of $-\ln (1-\varrho)$ vs $\psi$ 
(a)

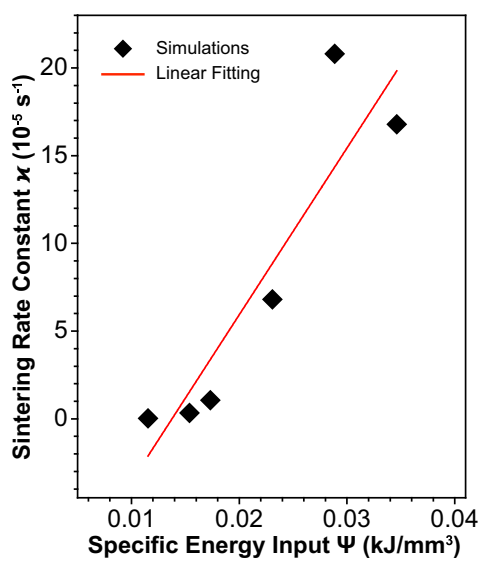

(b)

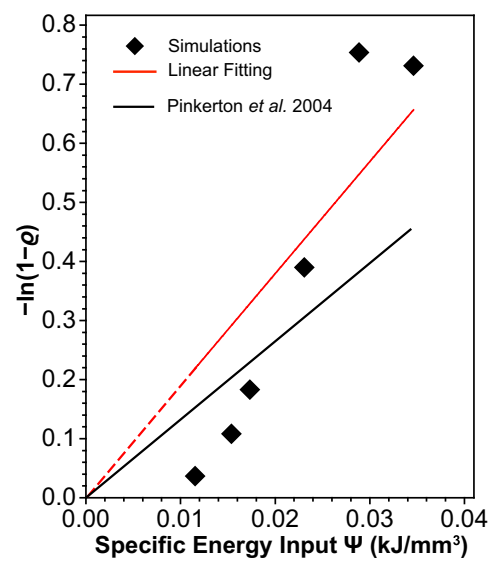

(c)

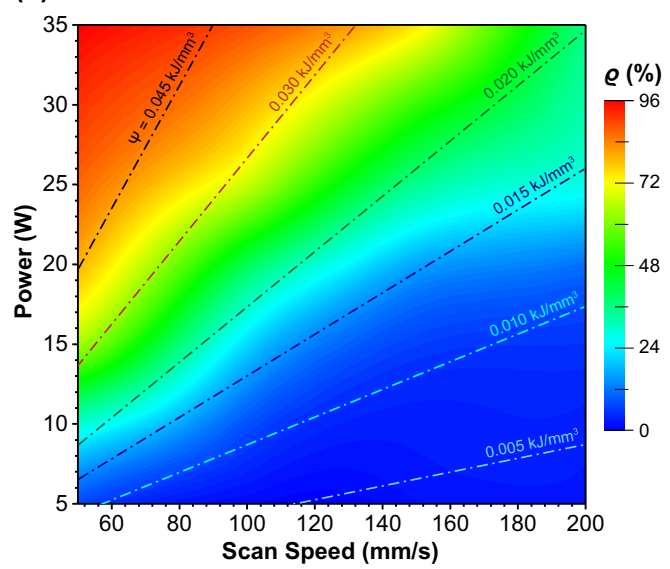

Fig. 7 a The sintering rate constant $\kappa$ and $\mathbf{b}-\ln (1-\varrho)$ as a function of the specific energy input $\Psi$. Linear fittings are illustrated by the red lines; c map of densification factor $\varrho$ along with different laser power and scan speed, where the dash-dotted lines denote different specific energy input $\Psi$

gives the densification coefficient $K=18.97$, as shown in Fig. $7 b$. It should be noted that $K$ is related to the material properties and the particle diameter distribution in the powder bed.19 The $K$ value obtained here is in line with the experimental $K=12.6$ according to refs ${ }^{19,62}$ where the SS316L particles have diameters $<45 \mu \mathrm{m}$. The agreement in $K$ shows the applicability of the developed model in simulating the microstructure densification. We also present a detailed map of the densification factor $Q$ in Fig. 7c. Following the isoline of the specific energy input $\psi$ (dashdotted lines in Fig. 7c), increasing laser power/scan speed actually leads to the increase of the densification $\varrho$. And when higher $\psi$ are kept, the faster increase of $\varrho$ can be found. It reveals another interesting fact that specific energy input may not be able to uniquely identify the densification of the processed component during SLS. A similar pattern has been experimentally observed in ref. 63

To sum up, in this work we perform 3D non-isothermal phasefield simulations to investigate the microstructure evolution during SLS. We recapitulate interesting phenomena during SLS, such as a high-gradient temperature field, mass transfer through partial melting, diffusion, and particle/grain necking and coarsening. We also reveal the influences of the processing parameters (i.e. laser power and scan speed) on microstructural features, including the porosity, surface morphology, temperature profile, particle/grain geometry, and densification. We further present the feasibility of the first-order kinetics for transient porosity during densification of the processed powder bed, and verify the linkage of the sintering rate constant and the densification factor to the specific energy input. Further investigations of the influence of factors such as the scan strategies, initial particle size distribution, and shape during SLS processing are expected in the near future.

\section{METHODS}

Powder bed generation using discrete element method

To generate the initial powder bed, the discrete element method (DEM) simulator YADE is used. ${ }^{64}$ The spheres are firstly created as the gaseous loose-packing cluster with no contacts between particles. The distribution of the particle diameter is shown in Supplementary Fig. 3a. Then, a gravitational force is imposed on the particles to make them spread into a rectangular box. The iterations continue until the deviation from force equilibrium on the particles is below a certain threshold, i.e, the particles are stationary. This process is shown in Supplementary Fig. 3b. After the powder bed generation, the center coordinate $\mathbf{r}_{O}$ and radius $R$ of each unique particle is recorded as a vertex $v_{i}\left(\mathbf{r}_{O}, R\right)$, and added into the vertices list $\mathbb{V}\left\{\nu_{i}\right\}$ for further optimization.
Implementation of finite element method

The model is numerically implemented by finite element method (FEM) within the program "NIsOS", developed by authors based on MOOSE framework. ${ }^{65}$ Eight-node hexahedron Lagrangian elements are chosen to mesh the geometry. Cahn-Hilliard equation in Eq. (11) is solved in a split way. ${ }^{66-68}$ Transient solver with preconditioned Jacobian-Free Newton-Krylov method (PJFNK) and backward Euler algorithm has been employed to solve the non-isothermal phase-field problems. Adaptive meshing and time stepping schemes are used to reduce the computation costs. The constraint of the order parameters is fulfilled using the penalty method. More details about the FEM implementation are shown in Supplementary Note 4.

\section{Parallel CPU computation}

The large-scale parallel CPU computations for each simulation domain, which has DOFs on the order of 10,000,000 for both nonlinear system and auxiliary system, are performed with 150 processors and 2 GByte RAM per processor based on OpenMPI. Each simulation consumes on the order 10,000 of CPU core*hours.

\section{DATA AVAILABILITY}

The authors declare that the data supporting the findings of this study are available within the paper and its Supplementary Information files.

\section{ACKNOWLEDGEMENTS}

The support from the European Research Council (ERC) under the European Union's Horizon 2020 research and innovation programme (grant agreement No 743116), German Research Foundation (DFG), the Profile Area From Material to Product Innovation - PMP and Open Access Publishing Fund of Technische Universität Darmstadt is acknowledged. M.Y. acknowledges the support from the 15th Thousand Youth Talents Program of China, the Research Fund of State Key Laboratory of Mechanics and Control of Mechanical Structures (MCMS-I-0419G01), and a project funded by the Priority Academic Program Development of Jiangsu Higher Education Institutions. We also greatly appreciate the access to the Lichtenberg HighPerformance Computer and the technique supports from the HHLR, Technische Universität Darmstadt.

\section{AUTHOR CONTRIBUTIONS}

Y.Y. performed the phase-field simulations, data processing, and manuscript writing. O.R. performed the powder bed generation and contributed to the manuscript writing. Y.B. supported numerical calculations in HPC. B.-X.X. supervised the project, analyzed the results, and revised the manuscript. M.Y. contributed by intensive consultation and discussion. All authors reviewed and approved the manuscript. 


\section{ADDITIONAL INFORMATION}

Supplementary information accompanies the paper on the npj Computational Materials website (https://doi.org/10.1038/s41524-019-0219-7).

Competing interests: The authors declare no competing interests.

Publisher's note: Springer Nature remains neutral with regard to jurisdictional claims in published maps and institutional affiliations.

\section{REFERENCES}

1. Kumar, S. Selective laser sintering: a qualitative and objective approach. JOM $\mathbf{5 5}$ 43-47 (2003).

2. Kruth, J.-P., Leu, M.-C. \& Nakagawa, T. Progress in additive manufacturing and rapid prototyping. CIRP Ann. 47, 525-540 (1998)

3. Khaing, M., Fuh, J. \& Lu, L. Direct metal laser sintering for rapid tooling: processing and characterisation of eos parts. J. Mater. Process. Technol. 113, 269-272 (2001).

4. Karapatis, N., Van Griethuysen, J. \& Glardon, R. Direct rapid tooling: a review of current research. Rapid Prototyp. J. 4, 77-89 (1998).

5. Gu, D., Meiners, W., Wissenbach, K. \& Poprawe, R. Laser additive manufacturing of metallic components: materials, processes and mechanisms. Int. Mater. Rev. 57, 133-164 (2012)

6. Gusarov, A., Yadroitsev, I., Bertrand, P. \& Smurov, I. Model of radiation and heat transfer in laser-powder interaction zone at selective laser melting. J. Heat Transf. 131, 072101 (2009).

7. Gusarov, A. \& Kruth, J.-P. Modelling of radiation transfer in metallic powders at laser treatment. Int. J. Heat Mass Transf. 48, 3423-3434 (2005).

8. Kruth, J.-P., Mercelis, P., Van Vaerenbergh, J., Froyen, L. \& Rombouts, M. Binding mechanisms in selective laser sintering and selective laser melting. Rapid Prototyp. J. 11, 26-36 (2005).

9. Li, J. Q., Fan, T. H., Taniguchi, T. \& Zhang, B. Phase-field modeling on laser melting of a metallic powder. Int. J. Heat Mass Transf. 117, 412-424 (2018).

10. Sing, S. L. et al. Direct selective laser sintering and melting of ceramics: a review. Rapid Prototyp. J. 23, 611-623 (2017).

11. Ko, S. H. et al. All-inkjet-printed flexible electronics fabrication on a polymer substrate by low-temperature high-resolution selective laser sintering of metal nanoparticles. Nanotechnology 18, 345202 (2007).

12. Williams, J. M. et al. Bone tissue engineering using polycaprolactone scaffolds fabricated via selective laser sintering. Biomaterials 26, 4817-4827 (2005).

13. Eshraghi, S. \& Das, S. Mechanical and microstructural properties of polycaprolactone scaffolds with one-dimensional, two-dimensional, and threedimensional orthogonally oriented porous architectures produced by selective laser sintering. Acta Biomater. 6, 2467-2476 (2010).

14. Tan, K. et al. Selective laser sintering of biocompatible polymers for applications in tissue engineering. Biomed. Mater. Eng. 15, 113-124 (2005).

15. Berry, E. et al. Preliminary experience with medical applications of rapid prototyping by selective laser sintering. Med. Eng. Phys. 19, 90-96 (1997).

16. Guo, D., Li, L.-t, Cai, K., Gui, Z.-I \& Nan, C.-w Rapid prototyping of piezoelectric ceramics via selective laser sintering and gelcasting. J. Am. Ceram. Soc. 87, 17-22 (2004).

17. Jhong, K. J. \& Lee, W. H. Fabricating soft maganetic composite by using selective laser sintering. In: 2016 IEEE International Conference on Industrial Technology (ICIT), 1115-1118 (IEEE, Taipei, Taiwan, 2016).

18. Deckard, C. R. \& Beaman, J. J. Recent advances in selective laser sintering. In: 14th conference on production research and technology, 447-452 (1987).

19. Simchi, A. Direct laser sintering of metal powders: Mechanism, kinetics and microstructural features. Mater. Sci. Eng. A 428, 148-158 (2006).

20. Olakanmi, E. Ot, Cochrane, R. \& Dalgarno, K. A review on selective laser sintering/ melting $(\mathrm{s} / \mathrm{s} / \mathrm{s} / \mathrm{m})$ of aluminium alloy powders: processing, microstructure, and properties. Progr. Mater. Sci. 74, 401-477 (2015).

21. Dong, L., Makradi, A., Ahzi, S. \& Remond, Y. Three-dimensional transient finite element analysis of the selective laser sintering process. J. Mater. Process. Technol. 209, 700-706 (2009).

22. Arsoy, Y. M., Criales, L. E. \& Özel, T. Modeling and simulation of thermal field and solidification in laser powder bed fusion of nickel alloy in625. Optics Laser Technol. 109, 278-292 (2019).

23. Ganeriwala, R. \& Zohdi, T. I. Multiphysics modeling and simulation of selective laser sintering manufacturing processes. Procedia CIRP 14, 299-304 (2014).

24. Gusarov, A., Laoui, T., Froyen, L. \& Titov, V. Contact thermal conductivity of a powder bed in selective laser sintering. Int. J. Heat Mass Transfer 46, 1103-1109 (2003).

25. Yi, M., Xu, B.-X. \& Gutfleisch, O. Computational study on microstructure evolution and magnetic property of laser additively manufactured magnetic materials. Comput. Mech. 64, 1-19 (2019).
26. Liu, F., Zhang, Q., Zhou, W., Zhao, J. \& Chen, J. Micro scale $3 \mathrm{~d}$ fem simulation on thermal evolution within the porous structure in selective laser sintering. J. Mater. Process. Technol. 212, 2058-2065 (2012).

27. Ganeriwala, R. \& Zohdi, T. I. A coupled discrete element-finite difference model of selective laser sintering. Granul. Matter 18, 21 (2016).

28. Gusarov, A. \& Smurov, I. Modeling the interaction of laser radiation with powder bed at selective laser melting. Phys. Procedia 5, 381-394 (2010).

29. Xiao, B. \& Zhang, Y. Numerical simulation of direct metal laser sintering of singlecomponent powder on top of sintered layers. J. Manuf. Sci. Eng. 130, 041002 (2008).

30. Zohdi, T. I. Modeling and simulation of laser processing of particulatefunctionalized materials. Arch. Comput. Methods Eng. 24, 89-113 (2017).

31. Wang, X. C. et al. Direct selective laser sintering of hard metal powders: experimental study and simulation. Int. J. Adv. Manuf. Technol. 19, 351-357 (2002).

32. Agarwala, M., Bourell, D., Beaman, J., Marcus, H. \& Barlow, J. Direct selective laser sintering of metals. Rapid Prototyp. J. 1, 26-36 (1995).

33. Olakanmi, E. Selective laser sintering/melting (sls/s/m) of pure al, al-mg, and al-si powders: effect of processing conditions and powder properties. J. Mater. Process. Technol. 213, 1387-1405 (2013).

34. Klocke, F. \& Wagner, C. Coalescence behaviour of two metallic particles as base mechanism of selective laser sintering. CIRP Ann. 52, 177-180 (2003).

35. Keller, T. et al. Application of finite element, phase-field, and calphad-based methods to additive manufacturing of ni-based superalloys. Acta Mater. 139, 244-253 (2017).

36. Wang, S.-L. et al. Thermodynamically-consistent phase-field models for solidification. Phys. D Nonlinear Phenom. 69, 189-200 (1993).

37. Kazaryan, A., Wang, Y. \& Patton, B. R. Generalized phase field approach for computer simulation of sintering: incorporation of rigid-body motion. Scr. Mater. 41, 487-492 (1999)

38. Ahmed, K., Yablinsky, C., Schulte, A., Allen, T. \& El-Azab, A. Phase field modeling of the effect of porosity on grain growth kinetics in polycrystalline ceramics. Model. Simul. Mater. Sci. Eng. 21, 065005 (2013).

39. Ahmed, K., Pakarinen, J., Allen, T. \& El-Azab, A. Phase field simulation of grain growth in porous uranium dioxide. J. Nucl. Mater. 446, 90-99 (2014).

40. Millett, P. C. et al. Phase-field simulation of intergranular bubble growth and percolation in bicrystals. J. Nucl. Mater. 425, 130-135 (2012).

41. Zhang, X. \& Liao, Y. A phase-field model for solid-state selective laser sintering of metallic materials. Powder Technol. 339, 677-685 (2018).

42. Lu, L.-X., Sridhar, N. \& Zhang, Y.-W. Phase field simulation of powder bed-based additive manufacturing. Acta Mater. 144, 801-809 (2018)

43. Yang, Y., Yi, M., Xu, B.-X. \& Chen, L.-Q. Phase-field modeling of non-isothermal grain coalescence in the unconventional sintering techniques. Prepring at https://arxiv.org/abs/1806.02799v1 (2018).

44. Penrose, O. \& Fife, P. C. Thermodynamically consistent models of phase-field type for the kinetic of phase transitions. Phys. D Nonlinear Phenom. 43, 44-62 (1990).

45. Moelans, N., Blanpain, B. \& Wollants, P. An introduction to phase-field modeling of microstructure evolution. Calphad 32, 268-294 (2008).

46. Wang, Y. U. Computer modeling and simulation of solid-state sintering: a phase field approach. Acta Mater. 54, 953-961 (2006).

47. Cahn, J. W. \& Hilliard, J. E. Free energy of a nonuniform system. i. interfacial free energy. J. Chem. Phys. 28, 258-267 (1958).

48. Ruelle, D. Statistical Mechanics: Rigorous Results (World Scientific, Singapore, 1999).

49. German, R. in Sintering of Advanced Materials, 3-32 (Elsevier, Oxford, UK, 2010).

50. Landau, L. D. \& Lifshitz, E. M. Statistical Physics: V. 5: Course Of Theoretical Physics (Pergamon Press, Oxford, UK, 1968).

51. Asp, K. \& Åren, J. Phase-field simulation of sintering and related phenomena-a vacancy diffusion approach. Acta Mater. 54, 1241-1248 (2006).

52. Gugenberger, C., Spatschek, R. \& Kassner, K. Comparison of phase-field models for surface diffusion. Phys. Rev. E 78, 016703 (2008).

53. Fan, D. \& Chen, L.-Q. Computer simulation of grain growth using a continuum field model. Acta Mater. 45, 611-622 (1997).

54. Kim, S. G., Kim, D. I., Kim, W. T. \& Park, Y. B. Computer simulations of twodimensional and three-dimensional ideal grain growth. Phys. Rev. E 74, 061605 (2006)

55. Krill, C. \& Chen, L.-Q. Computer simulation of 3-d grain growth using a phase-field model. Acta Mater. 50, 3059-3075 (2002).

56. Vedantam, S. \& Patnaik, B. S. V. Efficient numerical algorithm for multiphase field simulations. Phys. Rev. E 73, 016703 (2006).

57. Permann, C. J., Tonks, M. R., Fromm, B. \& Gaston, D. R. Order parameter remapping algorithm for $3 \mathrm{~d}$ phase field model of grain growth using fem. Comput. Mater. Sci. 115, 18-25 (2016).

58. Welsh, D. J. \& Powell, M. B. An upper bound for the chromatic number of a graph and its application to timetabling problems. The Comput. J. 10, 85-86 (1967).

59. McGuire, M. Austenitic Stainless Steels, 406-410 (Elsevier, Oxford, UK, 2001). 
60. Moelans, N., Blanpain, B. \& Wollants, P. Quantitative analysis of grain boundary properties in a generalized phase field model for grain growth in anisotropic systems. Phys. Rev. B 78, 024113 (2008).

61. German, R. Sintering: From Empirical Observations To Scientific Principles (Butterworth-Heinemann, Waltham, MA, UK, 2014).

62. Pinkerton, A. J. \& Li, L. The behaviour of water-and gas-atomised tool steel powders in coaxial laser freeform fabrication. Thin Solid Films 453, 600-605 (2004).

63. Prashanth, K., Scudino, S., Maity, T., Das, J. \& Eckert, J. Is the energy density a reliable parameter for materials synthesis by selective laser melting? Mater. Res. Lett. 5, 386-390 (2017).

64. Šmilauer, V. et al. Yade Documentation 2nd edn. https://yade-dem.org/doc/Yade. pdf (2019).

65. Tonks, M. R., Gaston, D., Millett, P. C., Andrs, D. \& Talbot, P. An object-oriented finite element framework for multiphysics phase field simulations. Comput. Mater. Sci. 51, 20-29 (2012).

66. Elliott, C. M., French, D. A. \& Milner, F. A second order splitting method for the cahn-hilliard equation. Numer. Math. 54, 575-590 (1989).

67. Zhao, Y., Stein, P. \& Xu, B.-X. Isogeometric analysis of mechanically coupled cahnhilliard phase segregation in hyperelastic electrodes of li-ion batteries. Comput. Methods Appl. Mech. Eng. 297, 325-347 (2015).
68. Balay, S., Gropp, W. D., Mclnnes, L. C. \& Smith, B. F. in Modern software tools for scientific computing, 163-202 (Springer, New York, USA, 1997).

cC) (i) Open Access This article is licensed under a Creative Commons Attribution 4.0 International License, which permits use, sharing, adaptation, distribution and reproduction in any medium or format, as long as you give appropriate credit to the original author(s) and the source, provide a link to the Creative Commons license, and indicate if changes were made. The images or other third party material in this article are included in the article's Creative Commons license, unless indicated otherwise in a credit line to the material. If material is not included in the article's Creative Commons license and your intended use is not permitted by statutory regulation or exceeds the permitted use, you will need to obtain permission directly from the copyright holder. To view a copy of this license, visit http://creativecommons. org/licenses/by/4.0/.

(c) The Author(s) 2019 